\title{
Gold in Electrical Contacts
}

\section{CURRENT PRACTICES AND FUTURE DEVELOPMENTS}

\author{
Morton Antler \\ Bell Telephone Laboratories, Columbus, Ohio
}

This review describes the characteristics of gold and gold alloys that make them suitable for electrical contacts in many types of circuits. Current practices in the use of gold-based materials for relay, slip ring, and connector applications are summarised, and possible trends in the future development of gold for contacts are considered.

Gold and its alloys are among the most common of contact metals. They are used in a variety of types of separable contacts for low and medium energy circuits, including relay make-break contacts, slip ring sliding contacts, and in connectors. Also, the nobility, softness, ductility, and high adhesion of pure gold make it an ideal material for permanent joints by thermocompression bonding in semiconductor devices.

The major portion of industrial gold used in the United States is for electrical contacts. In 1968, this amounted to approximately 25 tons $(800,000$ troy ounces), and it is predicted that this level will increase 50 per cent by 1973 . The world consumption of gold for contacts can be assumed to be three times this amount, based on known usages of gold in the electronic industries of major consuming countries and on estimates from the U.S.S.R.

The popularity of gold originates in its unique chemical stability; it is the least reactive metal. Electrical contacts for low voltage applications are not able to cope with more than minimal amounts of insulating oxide, tarnish, or (for platinum group metals) friction polymer. This is because it is not possible electrically to puncture insulating films to establish metal-metal contact at low voltages. Also, the requirement of easy separability makes it impractical to design contacts for the large deformations
Fig. 1 Contacts in sealed reed relays may be of silver-gold alloys or may be electroplated. This illustration shows a relay of this type in which the gold-plated reeds (left) are sealed in an inert atmosphere (centre) and enclosed within an activating coil. The relay (right) has three separate contact pairs in a single eoil.
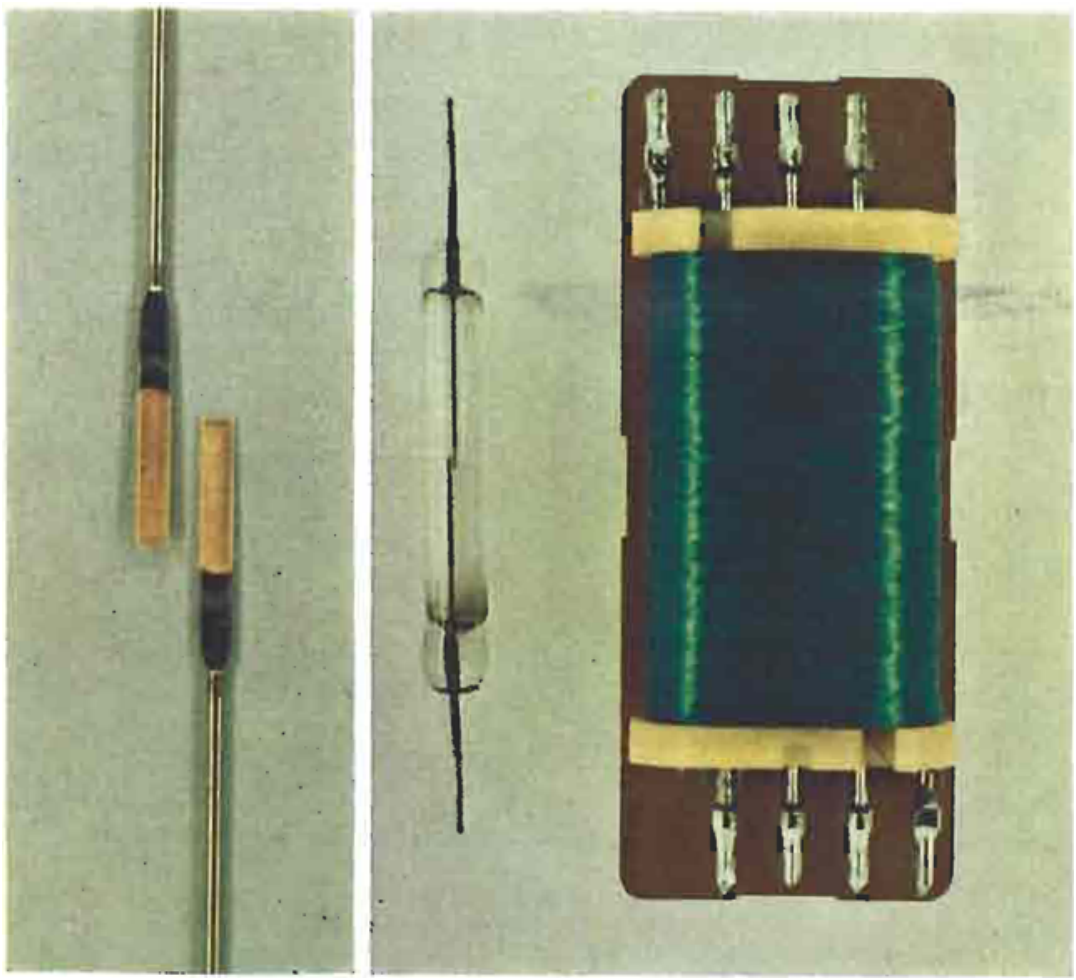

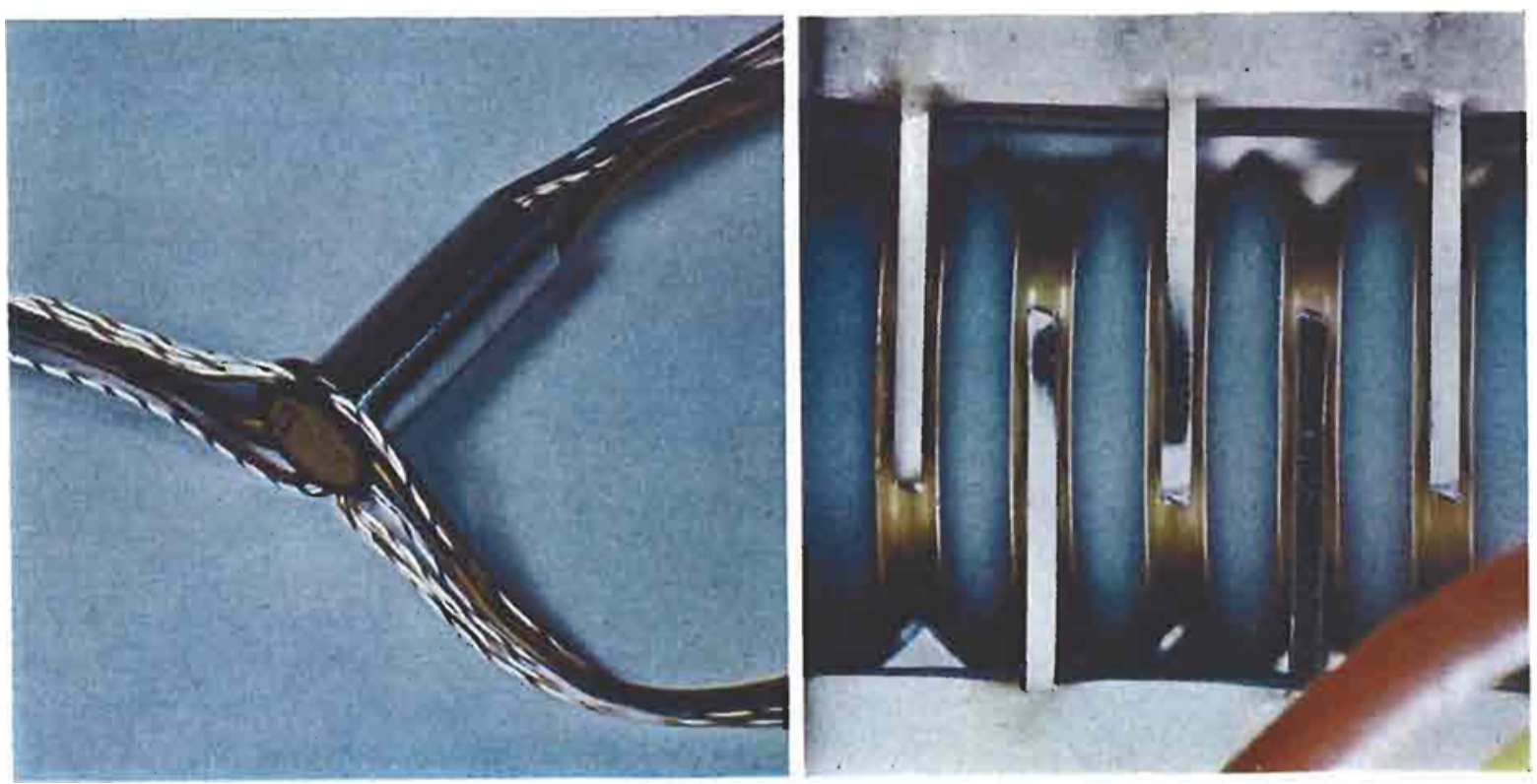

Fig. 2 An instrument slip ring assembly by Litton Industries, showing the gold-plated slip ring and the alloy gold brusl contacts on the right.

produced by high loads-which can mechanically break films to establish a viable contact.

The rapid development of low voltage solid state devices and their growing applications in computers, communications systems and instrumentation is responsible for the increase in the utilisation of gold for contacts. The moderate cost of the metal compared to that of the components in which it is used, and its availability in unlimited amount at relatively constant price, also have been responsible for stimulating the use of gold for contacts.

This review concerns gold in electrical contacts: its forms, properties, current practices, and discusses possible future developments in gold-based contact technology.

\section{Forms of Gold}

Gold is used on contacts in both wrought form and as electrodeposits. Weldments on the ends of the spring members of relay and of connector contacts are usually 18 carat, such as the gold-silver alloy. Cladded gold alloy "stripes" on sheet materials, such as copper-based spring metals, which are subsequently blanked and formed to shape, are also growing in importance. These solid golds are localised at the mating site of the contact member and reduce the amount that would otherwise be necessary. Monolithic gold alloy contacts are also employed in slip ring-brush systems.

Electrodeposits are by far the most common form of gold on contacts, because of the ease by which they can be obtained significantly thinner than is possible by welding or cladding. Platings are generally 99.0 to 99.9 per cent gold, with the balance a second metal for hardening, such as cobalt, nickel, silver or cadmium. Pure gold in separable contacts is soft, and wears poorly.

Many plating solutions have been devised which produce acceptable electrodeposits for contacts. Gold is deposited on small members by barrel methods, while rack plating is feasible for large components, and is used for small contacts when the deposits are to be applied selectively by partial immersion in the plating solution. Recently, jet plating, in which a stream of gold-containing electrolyte is made to impinge on an uncovered area of partially masked surface, has been applied to some elements.

Other coating methods for gold are relatively little used on contacts. These include electroless deposition and the physical deposition techniques, such as sputtering and ion plating.

\section{Relay Contacts}

Relays establish or break active electrical circuits. Contact is made by butting surfaces at a few grams pressure. The desirable characteristics of a material are nobility to assure low contact resistance, minimal adhesion of its surfaces to permit contact separation, and little tendency to erosive wear. Pure gold is not satisfactory, because of strong adhesion and poor erosion characteristics. However, at moderate current and voltages, say below 5 amperes at 50 volts, gold alloys have been developed which display a suitable combination of qualities to be successful in some applications. The maximum tolerable power level depends also on the size of the contacts and on their required service life. Relays having 


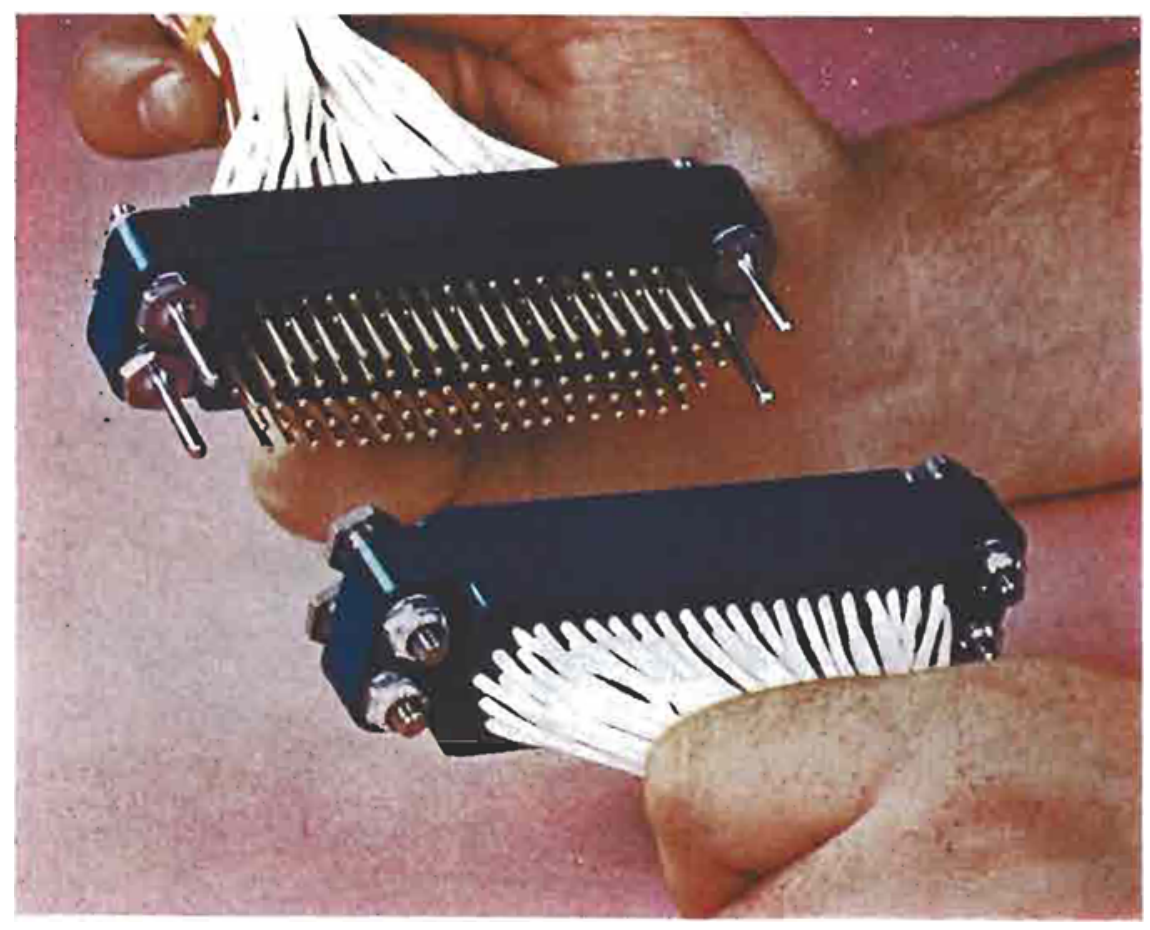

Fig. 3 A rack and panel connector with gold-plated pin-socket contaets, manufactured by Burndy Corpora. tioll.

contacts exposed to the air or sealed with the actuating coil in a glass or metal envelope often have contacts made of 75 gold- 25 per cent silver, or 69 gold -25 silver-6 per cent platinum, several thousandths of a centimetre thick. Platings of silver-, nickel-, or cobalt-hardened gold in similar thicknesses are also common.

A major advance in relay contact materials for telephone applications involves the use of a composite of about 20 micrometres of a 22 carat hard gold alloy over palladium on one contact. The other member is solid palladium. This system resists both erosive wear and sliding damage. If the contact involves arcing the gold is quickly lost, leaving palladium which has excellent erosive characteristics. If the contact is used where rubbing predominates, the gold reduces the formation of friction polymer.

The dry reed relay is widely used for fast switching at low (0.5 ampere) current, and particularly at millivolt-milliampere levels. It involves a structure having strap-like magnetic nickel-iron alloy spring members, sealed in a glass envelope with an inert or reducing atmosphere (Fig. 1). A coil surrounds the envelope and, when actuated, the relay can perform switching functions, such as make, break, or transfer of current between fixed members in multicontact systems. The contact is made by plating several micrometres of a hard gold on the ends of the reeds. Sometimes, the contact is heated to diffuse the gold into the substrate, thus producing a surface with little sticking tendency. A dual plating of silver and a pure gold top plate is also used for diffused contacts.
In high energy switching, freedom from insulating films is not so essential; however, contact deterioration due to electrical erosion is correspondingly more important, and non-gold contacts are employed.

\section{Sliding Contacts}

Many sliding contacts carry current continuously. Typical forms which use gold are instrument slip rings and brushes such as those shown in Fig. 2. Sliding gold contacts in switches and encoders may break low energy circuits. In addition to freedom from films, essential for low contact resistance and minimal electrical noise, sliding contacts must also operate with low friction and negligible wear. Instrument slip rings are usually electroplated with 2.5 to 12.5 micrometres of low alloy gold hardened with nickel or cobalt. Electroformed pure gold, machined to finish dimensions, is used as a substrate for some high reliability slip rings. Wrought alloys, such as 90 gold-10 copper, 75 gold-22 silver3 nickel, and 69 gold-25 silver-6 platinum are also common ring materials. Brushes are generally monolithic and have a high elastic limit, in addition to nobility and good sliding behaviour. A widely used brush material has the composition, 71.5 gold-14.5 copper-8.5 platinum-4.5 silver-1.0 zinc. Sliding contacts are often coated with a thin film of a synthetic lubricant to reduce sliding wear.

\section{Connector Contacts}

Connectors are designed into electrical packages to permit assembly from smaller units and to facilitate circuit changes and repairs. Connector contacts 
carry current when mated; only rarely are they required to make or break active circuits. Connectors with gold contacts are often used when voltages are below 50, current is below 10 amperes, and when the connector must serve in long life high reliability applications, especially in polluted atmospheres. A typical connector is shown in Figure 3. Again, freedom from films makes gold a preferred contact material. Sticking and wear also limit connector life, the former because of the lengthy periods contacts may remain mated, and the latter because of the much higher loads, typically 50 to 250 grams, of connector contacts compared to those in relays and in slip rings. Connectors with gold plated contacts are widely used in computer, telephone, and instrument applications.

A major category of connectors is the type which mates to the gold-plated contact fingers on the edges of printed circuit boards (Fig. 4). The golds most widely used in connector contacts are hard low alloy deposits in thicknesses from 0.5 to 5 micrometres. Substrates are usually copper alloys-brass, phosphor bronze, and beryllium copper. There is also some use of nickel and Kovar substrates. Printed circuit board substrates for edge-type contacts are pure copper foil. Nickel underplate is frequently specified as a barrier to diffusion in service for extended times above $60^{\circ} \mathrm{C}$. Otherwise, elements from the substrate would pass through the gold to the surface and oxidise to detrimental films.

\section{Future Developments}

Developments over the next few years in gold technology for contacts probably will not involve radical changes in practices. Rather, improvements will be made along lines that have been fruitful in the past.

\section{Gold Electrodeposits}

Better gold plating solutions and procedures for using them will yield deposits that are less porous than today's golds. These platings will also have a reduced tendency to sliding wear and to erosion in switching applications. The tolerance of most solutions for impurities is small, and means may be found to improve this characteristic. Gold deposits alloyed with significant amounts of base metals are widely used in the jewellery industry, and these golds, and newer high alloy deposits, are likely to be adopted to a limited extent in connector contacts and in sliding contacts for cost reduction, compared to the higher purity golds. Such deposits will have to be examined for service in polluted atmospheres and at elevated ambient temperature; reduced nobility and a tendency to produce films by diffusion of base alloying elements to the surface are possible limitations of alloy golds.

\section{Improved Selective Plating Processes}

Selective processes for depositing gold only on the mating surfaces of contact members will continue
Fig. 4 This printed circuit is a character generation board for a visual display unit produced by International Computers Limited. It is typical of modern printed circuitry, incorporating MOS micro-integrated circuits. The connections at the left-hand side are gold plated to ensure reliable contact when the board is plugged in.

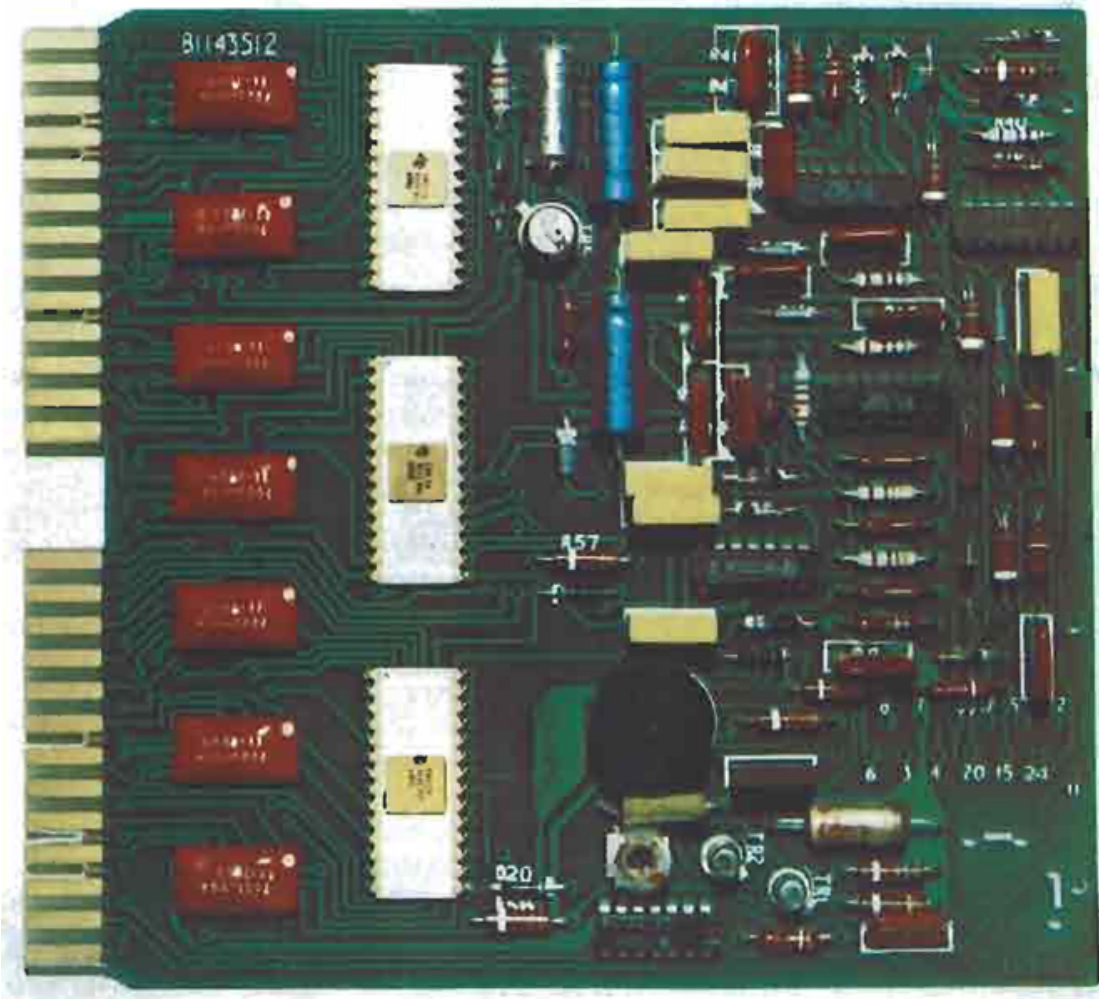


to be evolved. Some selective procedures now available are little used because the limited number of devices produced which can use them does not justify the tooling expense.

\section{Role of Substrate}

The composition, topography, and method of treatment of the substrate prior to gold plating, are important determinants of the properties of the deposit. Improved properties, such as in porosity and wear resistance, can be achieved by selection of underplates. Post-plate treatments, like the application of certain contact lubricants, can improve sliding performance and may reduce corrosion at pore sites. More careful selection and processing of substrate materials and post-plating treatments will become common for connector contacts.

Gold is indispensable for electrical contacts. The increase in its utilisation will parallel the growth of the electronics field.

\section{Organic Gold Compounds as Possible Lubricating Oil Additives}

\section{DEPOSITION OF METAL ON WEARING SURFACES}

To meet the lubrication problems presented by continual advances in the design and efficiency of automobile engines a wide range of chemical additives has been developed over the past thirty or more years. The amounts of these compounds now produced represents a considerable contribution to the whole chemical industry, and a great deal of research has been, and is still being, conducted by their producers. One of the major types of additives, the metal dialkylphosphorodithioates, has achieved wide and large-volume use as a corrosion inhibitor or anti-wear agent, and every leading oil company producing crankcase lubricants markets one or more products containing an additive of this kind - usually zinc dialkylphosphorodithioate - to the extent of a fraction of one per cent.

The precise mechanism by which these compounds reduce corrosion and wear has been investigated by a number of workers in the oil industry, but although it has been established that their effectiveness is directly related to their rate of thermal decomposition, the nature of the surface reaction is not yet fully understood. Efforts have therefore been concentrated on achieving optimum thermal stability by studying the effects of a number of alkyl groups as well as of changes in the metal cation upon both wear and coefficient of friction: It is believed, however, that the decomposition of the additive leads to the formation of a corrosion resistant film by reaction with the metal of the bearing surfaces, so reducing mechanical wear. This reaction naturally gives rise to a certain amount of chemical attack, and it has been one of the objectives of research in this field to develop an additive that would deposit a film of soft metal on the rubbing surfaces without involving them in a chemical reaction.

In the course of an investigation aimed at the deposition of metal on to these surfaces, two members of the Central Research Division of Mobil Research and Development Corporation, Princeton, New Jersey, have now found that, by substituting gold for the generally used zinc as the cation in dialkylphosphorodithioates, a deposit of gold is formed. A preliminary report on this work was published in Nature (1971, 231, May 24th, 87-88) by Joseph J. Dickert and Carleton N. Rowe, while U.S. Patent 3,554,908 also discloses the nature of the development.

\begin{tabular}{|c|c|c|}
\hline \multicolumn{3}{|c|}{ Wear Rate of Steel Pin } \\
\hline $\begin{array}{c}\text { Time } \\
\text { (minutes) }\end{array}$ & $\begin{array}{c}\text { Total wear } \\
\left(\mathrm{cm}^{3} \times 10^{7}\right)\end{array}$ & $\begin{array}{c}\text { Average wear rate } \\
\left(\mathrm{cm}^{3} \mathrm{~cm}^{-1} \times 10^{12}\right)\end{array}$ \\
\hline 15 & 3.9 & 43.0 \\
30 & 5.6 & 30.0 \\
60 & 5.6 & 15.0 \\
300 & 7.8 & 4.2 \\
1000 & 8.7 & 1.5 \\
\hline
\end{tabular}

Using two types of wear testing apparatus, a pin-on-disc machine and a four-ball machine, they found that gold was deposited on the wear scars of the steel pins and of the steel balls from a solution of gold dineopentylphosphorodithioate in $n$-hexadecane. The gold films were clearly visible and were conclusively identified by X-ray diffraction. More importantly, the average wear rate was found to diminish with time and to become extremely small after only a short period of testing. An example of this effect is given in the table, which shows the average wear rate expressed as volume per unit sliding distance, using a separate pin for each experiment.

While the mechanism is not understood, the evidence suggests that the local surface temperature on the rubbing surfaces contributes to the deposition of the gold in the contact area, since no deposition was found to occur elsewhere. The gold was seen to form preferentially on the surface in constant contact - the one having the sustained high surface temperature the pin or the stationary balls. In effect the system appears to become one of steel sliding against gold, and the great importance of the work lies in the fact that any loss of gold brought about by wear is replenished by further deposition. Thus the system achieves a balance of film wear and film formation depending upon the severity of operating conditions, and with proper balance the bearing surface can survive for as long as the new additive is present in solution.

L. B. H. 